\title{
The Application of SEM-Based EDS Microanalysis to the Study of Congenital Dyserythropoietic Anaemia Type-1 (CDA-I)
}

\author{
Errin Johnson ${ }^{1}$, Patrick Trimby ${ }^{2 *}$, Caroline $\mathrm{Scott}^{3}, \mathrm{Jill} \mathrm{Brown}^{3}$, Christian Babbs ${ }^{3}$, Aude-Anais Olijnik ${ }^{3}$ \\ and Veronica Buckle ${ }^{3}$ \\ 1. Sir William Dunn School of Pathology, University of Oxford, Oxford, UK \\ 2. Oxford Instruments Nanoanalysis, High Wycombe, UK \\ 3. Weatherall Institute for Molecular Medicine, University of Oxford, Oxford, UK \\ * Corresponding author: pat.trimby@oxinst.com
}

Congenital dyserythropoietic anaemia type-1 (CDA-I) is a form of moderate to severe anaemia which produces other symptoms ranging from jaundice to skeletal defects [1]. CDA-I specifically disrupts the erythroid lineage, such that erythropoiesis is affected at the polychromatic erythroblast stage and less erythrocytes (red blood cells) are produced. CDA-I erythroblasts exhibit a characteristic 'spongey' heterochromatin nuclear phenotype at the TEM level, though why this occurs, and how it relates to the disruption of erythroid differentiation, is unclear. There are two proteins implicated in CDA-I: codanin1 , which is thought to be involved in histone delivery $[2,3]$ and C15ORF41, which possesses nuclease activity [4]. However, the distinct function(s) of these proteins are unknown. Elucidating their role(s) in the DNA transcription, replication and/or repair pathways could help to identify new biomarkers to screen for specific CDA-I drug treatments and also further our fundamental understanding of erythropoiesis.

TEM has proven to be an important tool in the diagnosis and study of CDA-I. We aimed to build on this by using SEM-based microanalysis techniques to provide novel insights into the mechanisms underlying CDA-I. Specifically we used windowless energy dispersive x-ray spectrometry (EDS) to characterize the composition of the heterochromatin inclusions.

Cultured CDA-I erythroblasts were prepared for EM using a standard chemical fixation, heavy metal staining and epoxy resin embedding procedure or by high pressure freezing and freeze substitution with uranyl acetate, followed by processing into acrylic resin. Ultrathin sections were initially imaged on a FEI Tecnai 12 TEM using a Gatan OneView camera. For EDS microanalysis, sections ranging from 70$500 \mathrm{~nm}$ in thickness were mounted onto either formvar or carbon film on copper grids and were not post-stained or coated prior to analysis. Grids were loaded into the STEM holder of either a Zeiss Merlin FEG-SEM or a Zeiss Sigma 300 FEG-SEM and imaged using the in-lens secondary electron detector (with inverted signal). For robust quantitative chemical analysis, we collected around 20 million x-rays per area at the whole cell, nucleus and sub-nucleus levels using either the Oxford Instruments X-Max $150 \mathrm{~mm}^{2}$ or windowless Ultim Xtreme detectors.

We have used EDS to quantify overall differences in the elemental composition of healthy nuclei compared to CDA-I nuclei, as well as to specifically characterize the ratio of nitrogen to phosphorus in the heterochromatin inclusions compared to the surrounding euchromatin and heterochromatin. This can help to determine whether these inclusions contain protein (high in nitrogen) or DNA (high in phosphorus). Extensive testing was first performed to determine optimum beam conditions, sample preparation and section thickness with new generation solid state EDS detectors. As expected, we found that the windowless Ultim Xtreme detector was significantly more sensitive to low energy x-rays than 
the X-Max $150 \mathrm{~mm}^{2}$ detector, producing phosphorus and nitrogen maps with higher signal to noise over similar collection times (approx. 10-15 mins), but using a much lower beam current. This meant that the section built up less charge and was therefore more stable over the collection period, improving our lateral resolution. Initial quantification using the Ultim Extreme detector suggests that CDA-I nuclei contains more of both nitrogen and phosphorus than healthy nuclei and that there is variation in the relative abundance of both these elements across the heterochromatin inclusions in CDA-I nuclei (Fig 1). This raises the possibility that these inclusions could comprise of a mix of euchromatin and heterochromatin with variable histone modifications and we are in the process of investigating this further.

The application of SEM-based EDS microanalysis to CDA-I research has enabled us to shed new light on the ultrastructural and elemental changes associated with this condition, highlighting the advantages of using a combined EM approach to study human disease. We are in the process of extending the EDS approach to include serial sectioning, in order to produce a 3D elemental map of CDA-I nuclei and better understand exactly how chromatin organization is altered at both the ultrastructural and compositional levels in CDA-I erythroblasts.

\section{References:}

[1] H. Tamary et al., British Journal of Haematology, 130, (2005) 628-634.

[2] O. Dgany et al., American Journal of Human Genetics, 71, (2002) 1467-1474.

[3] K. Ask et al., Embo Journal, 31, (2012), 3229-3229.

[4] C. Babbs et al., Haematologica, 98, (2013),1383-1387.

[5] The authors acknowledge the use of SEM / EDS facilities at the University of Manchester.
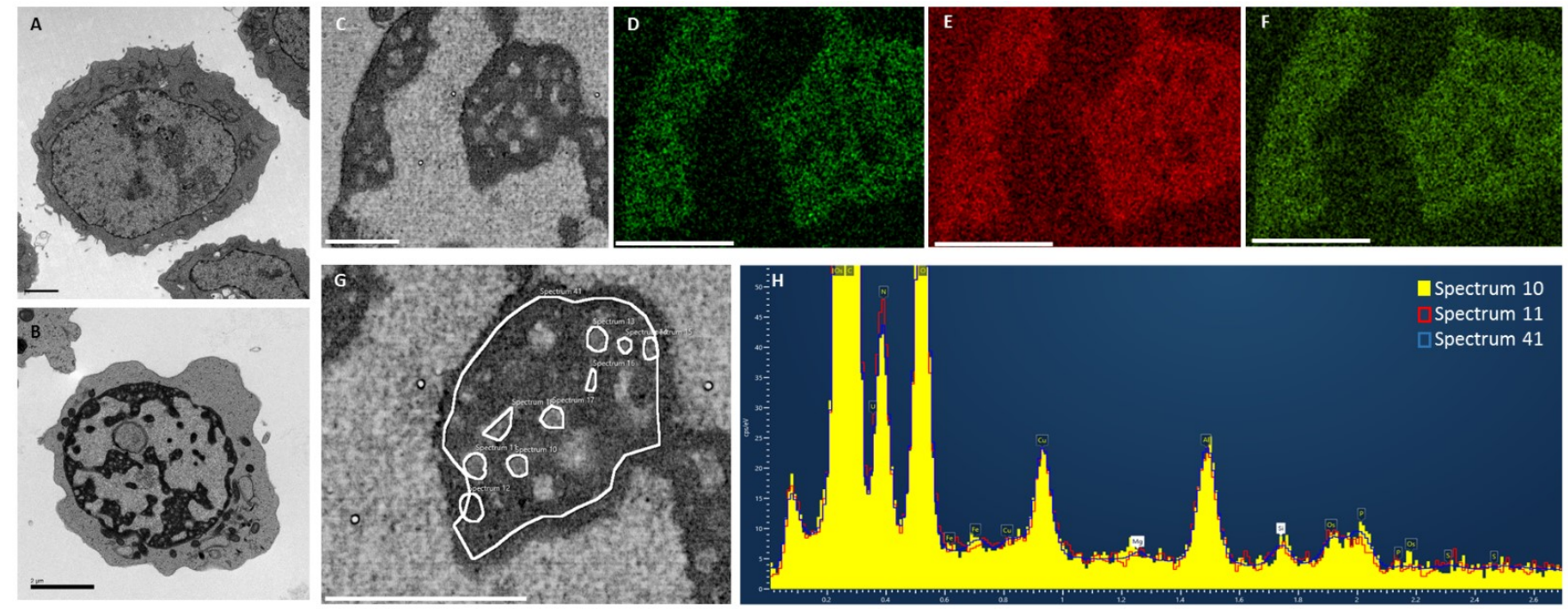

Figure 1. (A) TEM image of a healthy erythroblast cell. Scale 1um. (B) TEM image of a CDA-I erythroblast cell, exhibiting distinct holes / inclusions. Scale 2um. (C-F) EDS mapping results from part of a CDA-I nucleus showing SE image, $\mathrm{P}, \mathrm{N}$ and $\mathrm{U}$ distributions respectively. Scale 1um. $(\mathrm{G}-\mathrm{H})$ Analysis of extracted spectra from individual holes in an area of heterochromatin, indicating differences in $\mathrm{N}$ and $\mathrm{P}$ concentrations. Scale 1um. 